\title{
DUAL INTEGRAL EQUATIONS WITH TRIGONOMETRIC KERNELS
}

\author{
by J. S. LOWNDES \\ (Received 13th March 1965)
}

1. Consider the dual equations

$$
\begin{array}{ll}
\int_{0}^{\infty} \xi^{2 \lambda-1} \psi(\xi) \cos (\xi x) d \xi=f(x), & 0 \leqq x \leqq 1, \\
\int_{0}^{\infty} \psi(\xi) \cos (\xi x) d \xi=0, & x>1,
\end{array}
$$

where $-\frac{1}{2}<\lambda<\frac{1}{2}$.

A solution of these equations has been obtained by Srivastava (1) in the form of a Neumann series. In this note a formal solution for the equations (1) and (2) is obtained by a generalisation of a method due to Tranter (2) who obtained a solution for the special case when $\lambda=0$.

We shall use the representations ((3), pp. 48 and 170)

where $-\frac{1}{2}<\lambda<\frac{1}{2}$.

$$
\begin{aligned}
\frac{\sqrt{\pi}}{2} J_{\dot{\lambda}}(r \check{\zeta}) & =\frac{1}{\Gamma\left(\frac{1}{2}+\lambda\right)}\left(\frac{\xi}{2 r}\right)^{\lambda} \int_{0}^{r} \frac{\cos (\xi x)}{\left(r^{2}-x^{2}\right)^{\frac{1}{2}-\lambda}} d x, \\
& =\frac{1}{\Gamma\left(\frac{1}{2}-\lambda\right)}\left(\frac{2 r}{\xi}\right)^{\lambda} \int_{r}^{\infty} \frac{\sin (\xi x)}{\left(x^{2}-r^{2}\right)^{\frac{1}{2}+\lambda}} d x,
\end{aligned}
$$

Equations in which $\sin (\xi x)$ replaces $\cos (\xi x)$ in (1) and (2) are also solved.

2. Integrating equation (2) with respect to $x$ we find, as in (2), that

$$
\int_{0}^{\infty} \xi^{-1} \psi(\xi) \sin (\xi x) d \xi=0, \quad x>1
$$

Multiplying equations (1) and (5) respectively by $\frac{(2 r)^{-\lambda}}{\Gamma\left(\frac{1}{2}+\lambda\right)}\left(r^{2}-x^{2}\right)^{\lambda-\frac{1}{2}}$ and $\frac{(2 r)^{\lambda}}{\Gamma\left(\frac{1}{2}-\lambda\right)}\left(x^{2}-r^{2}\right)^{-\lambda-\frac{1}{2}}$ and integrating with respect to $x$ between $0, r$ and $r, \infty$, we find, using the representations (3) and (4), that

$$
\begin{aligned}
\frac{\sqrt{\pi}}{2} \int_{0}^{\infty} \xi^{\lambda-1} \psi(\xi) J_{\lambda}(\xi r) d \xi & =\frac{(2 r)^{-\lambda}}{\Gamma\left(\frac{1}{2}+\lambda\right)} \int_{0}^{r} \frac{f(x)}{\left(r^{2}-x^{2}\right)^{\frac{1-\lambda}{2}}} d x, \quad 0<r<1, \\
& =0, \quad r>1 .
\end{aligned}
$$


Applying the Hankel inversion theorem to equation (6) gives

$$
\sqrt{\pi} \xi^{\lambda-2} \psi(\xi)=\frac{2^{1-\lambda}}{\Gamma\left(\frac{1}{2}+\lambda\right)} \int_{0}^{1} r^{1-\lambda} J_{\lambda}(\xi r) d r \int_{0}^{r} \frac{f(x)}{\left(r^{2}-x^{2}\right)^{\frac{1}{2}-\lambda}} d x,
$$

as a solution of equations (1) and (2). When $\lambda=0$ this reduces to Tranter's solution.

3. To solve the similar pair of equations

$$
\begin{aligned}
& \int_{0}^{\infty} \xi^{2 \lambda-1} \psi(\xi) \sin (\xi x) d \xi=f(x), \quad 0 \leqq x \leqq 1, \\
& \int_{0}^{\infty} \psi(\xi) \sin (\xi x) d \xi=0, \quad x>1,
\end{aligned}
$$

where $-\frac{1}{2}<\lambda<\frac{1}{2}$, we first differentiate (8) with respect to $x$ and find

$$
\int_{0}^{\infty} \xi^{2 \lambda} \psi(\xi) \cos (\xi x) d \xi=f^{\prime}(x), \quad 0 \leqq x \leqq 1
$$

Operating on equations (10) and (9) in the same way as we have on equations (1) and (5) we get

$$
\begin{aligned}
\frac{\sqrt{\pi}}{2} \int_{0}^{\infty} \xi^{\lambda} \psi(\xi) J_{\lambda}(\xi r) d \xi & =\frac{(2 \boldsymbol{r})^{-\lambda}}{\Gamma\left(\frac{1}{2}+\lambda\right)} \int_{0}^{r} \frac{f^{\prime}(x)}{\left(r^{2}-x^{2}\right)^{\frac{1}{2}-\lambda}} d x, \quad 0<r<1, \\
& =0, \quad r>1 .
\end{aligned}
$$

Hence the solution of equations (8) and (9) is

$$
\sqrt{\pi} \xi^{\lambda-1} \psi(\xi)=\frac{2^{1-\lambda}}{\Gamma\left(\frac{1}{2}+\lambda\right)} \int_{0}^{1} r^{1-\lambda} J_{\lambda}(\xi r) d r \int_{0}^{r} \frac{f^{\prime}(x)}{\left(r^{2}-x^{2}\right)^{\frac{1}{2}-\lambda}} d x .
$$

\section{REFERENCES}

(1) K. N. SRivastava, Proc. Edin. Math. Soc. (4) 13 (1963), 351-354.

(2) C. J. Tranter, Proc. Edin. Math. Soc. (3) 13 (1963), 267-268.

(3) G. N. Watson, Theory of Bessel Functions (2nd edition, Cambridge University Press, 1944).

Department of Mathematics

UNIVERSITY OF STRATHCLYDE

Glasgow 